\title{
Intervención sobre las Funciones Ejecutivas (FE) desde el contexto educativo
}

\author{
Alexandra Pardos Véglia; María González Ruiz *
}

Resumen. Si bien la historia de la Neuroeducación es relativamente corta (sólo en la última década se comienza a aplicar al ámbito académico los métodos derivados de la neurociencia), el interés por esta disciplina es creciente y su eficacia para la mejora de aspectos importantes del contexto educativo parece demostrarse y concretarse cada vez más. Con el fin de comprender mejor los conceptos de las nuevas disciplinas como Neuroeducación o Neuropsicología infantil y, sobre todo, de aportar una propuesta práctica de aplicación de la Neurodidáctica en el aula, este artículo de revisión bibliográfica resume en primer lugar los conceptos básicos de estas disciplinas, para potenciar tanto el rendimiento académico como el ejecutivo en contexto educativo. Dentro del ámbito de la neuroeducación, las Funciones Ejecutivas (FE) constituyen un nuevo constructo que ha cobrado especial interés en los últimos años. Por ello, en un segundo lugar, este trabajo propone dos ejemplos de programas de intervención sobre las capacidades ejecutivas de metacognición y autorregulación, aplicables o adaptables al aula o a actividades educativas más amplias para niños de Educación Infantil y Primaria.

Palabras clave: neuropsicología infantil; neuroeducación; neurodidáctica, funciones ejecutivas; autorregulación; metacognición.

INTERVENÇÃO EM FUNÇÕES EXECUTIVAS (EF) NO CONTEXTO EDUCACIONAL Resumo. Embora a história da Neuroeducação seja relativamente recente (somente na última década que os métodos derivados da neurociência começaram a ser aplicados no âmbito acadêmico), o interesse por esta disciplina tem crescido muito e a sua eficácia na melhoria de aspectos importantes do contexto educacional tem sido comprovada e concretizada cada vez mais. Para entender melhor os conceitos de novas disciplinas como a Neuroeducação ou a Neuropsicologia infantil e, acima de tudo, para oferecer uma proposta prática de aplicação da Neurodidática em sala de aula, este artigo de revisão bibliográfica resume em primeiro lugar os conceitos básicos destas disciplinas, a fim de melhorar o desempenho acadêmico e executivo no contexto educacional. No âmbito da neuroeducação, as Funções Executivas (EF) constituem um novo conceito que ganhou interesse especial nos últimos anos. Portanto, em segundo lugar, este trabalho propõe dois exemplos de programas de intervenção sobre as capacidades executivas de metacognição e autorregulação, que são aplicáveis ou adaptáveis à sala de aula ou às atividades educativas mais abrangentes para as crianças da Educação Infantil e do Ensino Fundamental..

Palavras-chave: neuropsicologia infantil; neuroeducação; neurodidática; Funções Executivas; autorregulação; metacognição .

* Centro Neuropsicología Alexandra Pardos, España. 


\begin{abstract}
EXECUTIVE FUNCTION (EF) INTERVENTION IN EDUCATIONAL CONTEXT
Abstract. Interest in Neuroeducation is growing and its effectiveness improving aspects of the educational context seems to be demonstrated and made concrete more and more although the history of this discipline is relatively short (it is only in the last decade that the methods derived from neuroscience have been applied to the academic field). In order to better understand basic concepts from Neuroeducation or Child Neuropsychology and, above all, to provide a practical proposal for the application of Neurodidactics in the classroom, this bibliographic review article summarizes at a first moment the basic points of these disciplines, to enhance both, academic and executive performance, in the educational context. Neuroeducation is an area in which the Executive Functions constitute a new construct that has gained special interest in recent years. This paper proposes in a second part, two examples of intervention programs on metacognition and self-regulation capacities, applicable or adaptable to the classroom or to other educational activities for children in pre-school education and from 10 to 14 years old.
\end{abstract}

Keywords: child neuropsychology; neuroeducation; neurodidactics; executive functions; self-regulation; metacognition

\title{
1. INTRODUCCIÓN
}

La Neuropsicología estudia la relación entre la organización cerebral y la actividad cognitivo-conductual. En sus comienzos, con los estudios de Broca en el siglo XIX, se centró exclusivamente en los trastornos de la población adulta y sólo fue una década después -también partiendo de las dificultades del lenguaje- cuando amplió su foco de estudio a la población infantil (Ardila, Rosselli y Villaseñor, 2007). Actualmente, la neuropsicología infantil tiene como objetivo detectar disfunciones y lesiones cerebrales (surgidas a lo largo de la infancia, del embarazo o del parto) y llevar a cabo tratamientos enfocados a paliar las consecuencias de éstas.

La intervención neuropsicológica infantil se basa, como la de adultos, en la plasticidad cerebral, pero sobre todo en el principio de Kennard (1942), según el cual existe mayor recuperación neuronal cuando las lesiones se producen en edades tempranas (Ávila y Cuervo, 2010). Posteriormente y actualmente aún, numerosos autores han propuesto teorías sobre el desarrollo cognitivo en niños que permiten a la neuropsicología infantil establecerse como disciplina independiente de la neuropsicología adulta. Al ser la característica principal de la neuropsicología infantil tratar con un cerebro en constante desarrollo, el enfoque teórico que la sostiene es dinámico y global (Anderson, Northam y Wrennall, 2017) y tiene en cuenta tanto el desarrollo evolutivo (cognitivo, conductual y emocional) como los diferentes contextos en los que este desarrollo se produce (familiar, social y educativo). Además, la creciente 
importancia y necesidad de la neuropsicología infantil aumenta sus ámbitos de intervención, lo que se podría explicar también en parte, según Abad, Brusasca y Labiano (2009), por la aparición de nuevas poblaciones pediátricas (como los niños con cáncer o prematuros, entre otros) y la escolarización actual cada vez más temprana de los niños (en edades preescolares) que favorece la detección precoz de dificultades neuro-psicológicas, aumentando la preocupación e intervención socioeducativa por las patologías del sistema nervioso infantil (daño cerebral, trastornos por déficit atencional con o sin hiperactividad, dificultades de aprendizaje o trastornos psicomotores y del lenguaje). En general, para realizar un tratamiento neuropsicológico infantil eficaz, se recomienda evaluar o intervenir en las siguientes áreas específicas (Manga y Ramos, 1999, 2001): motricidad, praxias, percepción (en todas sus modalidades sensoriales), lenguaje, memoria (verbal, no verbal, a corto y largo plazo), cognición general (capacidad intelectual, de atención y FE) y aspectos psico-educativos o capacidades académicas en lectoescritura y aritmética, entre otros.

A menudo, también se requiere intervención con la familia o el contexto educativo. Además, los niños con lesiones cerebrales o déficits cognitivos tienden a presentar otro tipo de dificultades psicológicas asociadas, como problemas del estado de ánimo, ansiedad o baja autoestima (Anderson, Northam y Wrennall, 2017). Resulta por lo tanto necesario que la intervención clínica neuropsicológica infantil sea integrada, actuando sobre las funciones cognitivas, pero también sobre aspectos del área socio-emocional, Ilevando a cabo una comunicación y trabajo multidisciplinar con padres y profesores. En resumidas cuentas, puede afirmarse que la rehabilitación neuropsicológica tiene por objetivo conseguir mejoras funcionales, aportar estrategias que compensen las carencias y realizar cambios ambientales en función de las necesidades del paciente. Para conseguir estos objetivos, es necesario tanto el trabajo a nivel individual (realizar actividades que mejoren las capacidades cognitivas) como a nivel familiar y, en algunas ocasiones, interdisciplinar (pedagógico, socio-emocional, etc.).

Por otra parte, de la unión de la Educación con la Neuropsicología infantil surge la Neuroeducación que, como su nombre indica, aplica los conocimientos y las técnicas derivadas de la neurociencia al contexto educativo (Tapia y cols., 2017). Conocer mejor el funcionamiento del cerebro ayuda a mejorar la capacidad de aprendizaje y cada vez son más los educadores que lo tienen en cuenta a la hora de diseñar la programación educativa (Goswami, 2009). La neurociencia aporta conocimientos fundamentales acerca de las bases neurales del aprendizaje (tales como atención, memoria, razonamiento o lenguaje) y de otras funciones cerebrales, como las emociones o la conducta, que son, día tras día, estimuladas, evaluadas y fortalecidas y, a su vez, necesarias en el aula (Campos, 2010). En resumen, la Neuroeducación 
busca acercar al aula claves y herramientas para optimizar el aprendizaje de los alumnos y mejorar la preparación de los docentes (Bastién, Mora y Sánchez, 2013), basándose en la evidencia empírica. En este sentido, la Neurodidáctica ayuda a optimizar los procesos de enseñanza utilizando los conocimientos sobre el cerebro y recomienda a los docentes tener en cuenta los siguientes aspectos fundamentales propuestos por Tapia y cols. (2017):

- Conocer aspectos básicos del funcionamiento del cerebro humano y ser flexible en la metodología didáctica y de evaluación.

- Aprender a potenciar la atención durante el aprendizaje.

- Tener en cuenta la motivación, base del aprendizaje, y valorar tanto los procesos de aprendizaje (esfuerzo, actitud, evolución individual) como los resultados (notas).

- Llevar a cabo aprendizajes significativos y duraderos, en general más fáciles y eficaces que los puramente asociativos o memorísticos. Dejar a los niños el tiempo necesario para poder fijar y practicar los aprendizajes y adaptarse al ritmo de aprendizaje individual de cada niño.

- Valorar la importancia del entorno socio-emocional, del juego y del deporte.

- Reconocer las dificultades del aprendizaje de forma temprana para poder intervenir sobre ellas.

- Potenciar el autocontrol en el aula, habilidad fundamental para Ilevar a cabo aprendizajes eficaces. Se trata de un aspecto básico de las funciones ejecutivas, tratadas a continuación.

El conocimiento sobre las funciones cerebrales o cognitivas como percepción, atención, memoria, lenguaje, razonamiento entre otras, ha puesto de manifiesto su relación de sobra conocida hoy en día con los aprendizajes. En este contexto, destacan las Funciones Ejecutivas (FE), por haberse demostrado su relación con el rendimiento académico.

Las FE se pueden definir como la capacidad para ejercitar el autocontrol de la conducta o atención, mantener y manejar la información mentalmente, resolver problemas y adaptarse de manera flexible a cambios o situaciones inesperadas. Su importancia en el desarrollo infantil se ha ido haciendo cada vez más patente, ya que son aspectos cruciales para las actividades de la vida diaria, tanto en el ámbito académico como social. Concretamente, siguiendo a Anderson (2002), entre los principales elementos que incluyen las funciones ejecutivas, se encuentran la anticipación, la selección de objetivos, la planificación, el inicio de la actividad, la autorregulación, 
la flexibilidad mental y el control de la atención. Otros autores (Bell, 2013) también incluyen el autocontrol, la memoria de trabajo (capacidad de mantener información mental y utilizarla posteriormente) el control emocional o la inhibición de respuesta. Como puede deducirse, se trata de procesos que constituyen un papel fundamental no solo en el éxito cognitivo y académico, sino también en el emocional, comportamental y social. Actualmente, sólo se conoce la cronología aproximada del desarrollo de las funciones cognitivas (Zelazo y Müller, 2002): aparecen temprano en el desarrollo y presentan cambios importantes entre los 2 y 5 años. Pero parece haber acuerdo en que hay tres FE principales (control inhibitorio, memoria de trabajo y flexibilidad cognitiva) sobre las que se construyen las FE de orden superior, como el razonamiento, la resolución de problemas y la planificación (Diamond, 2014).

En cuanto a los correlatos neuro-funcionales, dado que las FE suelen entenderse como un conjunto de subprocesos indiferenciados e inter-conectados entre sí, resulta difícil asociarlas a una determinada área cerebral, pero globalmente se refieren al conjunto de funciones que dependen del lóbulo frontal y de sus conexiones con el resto de áreas. También se puede hacer una distinción entre FE "calientes" (con mayor carga afectiva), asociadas a la corteza orbitofrontal estrechamente relacionada con el sistema límbico y de FE "frías" (con mayor carga cognitiva), que se asocian a la corteza prefrontral dorsolateral (Hongwanishkul y cols., 2005).

Como se ha venido comentando, las tareas escolares requieren de muchos procesos y subprocesos incluidos en las funciones ejecutivas, tales como la habilidad de planificar el tiempo, organizar y priorizar información, revisar la ejecución, etc. (Meltzer 2018). La importancia de un buen funcionamiento ejecutivo es, por lo tanto, evidente para poder llevar a cabo aprendizajes, siendo éste un predictor fiable del rendimiento académico (Arias, 2012). En concreto, un estudio Ilevado a cabo por García y Muñoz (2000) pone de manifiesto la relación existente entre determinadas disfunciones ejecutivas y dificultades académicas. A continuación se resaltan algunas de las posibles repercusiones de las dificultades ejecutivas en el aula:

- Autorregulación o falta de inhibición: los niños con dificultades en esta área, tenderán a presentar comportamientos impulsivos y/o dispersos o excesiva inquietud motora ya que la falta de autorregulación puede darse a nivel conductual o cognitivo lo que resultará molesto en el ámbito académico y/o social y puede dificultar la adquisición de aprendizajes. Se trata de una habilidad especialmente afectada en niños con dificultades atencionales y/o de auto-control, ya que suelen perder el hilo de la tarea cuando se producen distractores externos. 
- Control emocional: en el ámbito escolar, son numerosas las situaciones en las que un niño debe afrontar situaciones negativas (discusión con un compañero, suspensos, frustración por dificultades de comprensión o mal rendimiento...), tratando de no presentar respuestas inadecuadas (ataques de ira, rabia, Ilantos o pataletas...). Los niños con peor control emocional, pueden presentar baja tolerancia a la frustración, desmotivación, ansiedad, tristeza, depresión, saltos de humor, pérdidas de control, rabietas, entre otros, en el entorno escolar, familiar y/o social.

- Flexibilidad cognitiva o cambio del foco atencional: esta capacidad permite modificar la conducta cuando sea necesario en función de las demandas externas, sin desconectar, y sus déficits pueden provocar dificultad para adaptarse a cambios con facilidad o rigidez cognitiva.

- Memoria de trabajo: se trata de la habilidad de mantener información mental y manipularla a corto plazo. Tiene un peso importante en los aprendizajes, para el seguimiento de consignas, la lecto-escritura o el cálculo mental. Suele estar afectada en alumno con dificultades de aprendizaje, a los que es necesario ayudar con estrategias y metodologías compensatorias.

- Monitorización: es la capacidad de supervisar si se realiza un plan o una ejecución de forma adecuada y de auto-ajustarse en el caso de que no sea así. En concreto, requiere que los alumnos sean conscientes de aquellos momentos en los que su respuesta no coincide con la prevista, y sean capaces de corregirla, por lo que un déficit en esta FE podría correlacionar con dificultad para la toma de conciencia y corrección de los propios errores, así como para llevar a cabo una generalización eficaz de los aprendizajes.

- Planificación/organización y toma de decisiones: organización y planificación son procesos mentales relacionados; el primero va desde la elaboración de un plan hasta la puesta en marcha de los medios para llevarlo a cabo, y el segundo puede definirse como la disposición de las acciones y el material que permitirán realizar el plan. Es clave dar una importancia real a este aspecto ejecutivo en los aprendizajes, hacer que los niños planifiquen la realización de las tareas de clase, organicen el estudio o aprendan a gestionar el tiempo. Un déficit en estas funciones implicaría necesidad de ayuda y supervisión externa para la gestión del tiempo y organización-planificación, tanto a nivel académico como en actividades de la vida diaria, las rutinas cotidianas de comidas o higiene. El niño que tenga dificultades en organización 
y planificación puede efectuar las tareas de manera impulsiva o desorganizada o lenta, equivocándose más que un niño que tenga esta habilidad desarrollada, necesitando de nuevo supervisión externa para poder concluir de forma eficaz.

En resumen, la creciente implementación -globalmente positiva- de tácticas neurodidácticas en el aula, con vistas a mejorar las FE y, en concreto, la capacidad de autorregulación y metacognición, implica la necesidad de llevar a cabo estrategias educativas que ayuden a los niños tanto a estimular funciones básicas para los aprendizajes como a mejorar su rendimiento académico. En caso de niños con dificultades cognitivas específicas, estas estrategias también servirán para minimizar las consecuencias de dichas dificultades. Todos los componentes de las FE son igualmente importantes y necesarios para el adecuado desarrollo global y rendimiento académico de los niños, pero, en adelante nos centraremos en el rol que desempeñan las capacidades de autorregulación y metacognición y en dos propuestas de intervención para potenciarlas en contexto educativo.

La autorregulación se puede definir como la capacidad que permite el control de la atención, del comportamiento (conducta y emoción) y de la acción dirigida a metas (Blair y Ursache, 2011). Se deduce que se trata de un concepto estrechamente relacionado con la actuación en el aula y con el rendimiento escolar. Numerosos estudios ponen de manifiesto los beneficios de presentar una adecuada autorregulación, correlacionando con mayor concentración, cooperación en el aula y mejores resultados académicos (Baron, Evangelou, Malmberg y Melendez-Torres, 2015). Además, se ha demostrado que la capacidad de autorregulación es susceptible de mejora (Barnett y cols., 2008; Diamond, Barnett, Thomas y Munro, 2007) y los resultados encontrados hasta ahora en el ámbito de la intervención en contexto clínico y académico parecen prometedores, especialmente en la primera infancia, por ser un periodo crítico del desarrollo durante el cual conseguir pequeños cambios puede ser muy beneficioso a largo plazo (Alexander, Entwisle, y Kabbani, 2001; O'Shaughnessy, Lane, Gresham, y Beebe-Frankenberger, 2003). En consecuencia, resulta crucial identificar prácticas educativas que fomenten el crecimiento de las capacidades de autorregulación en todos los niños y en particular en niños con necesidades específicas.

La bibliografía sobre autorregulación destaca, además, que determinadas poblaciones presentan mayor riesgo de dificultades en cuanto al autocontrol, concretamente en el caso de los niños preescolares (Baron, Evangelou, Malmberg y Melendez-Torres, 2017), de nivel socio-económico bajo (Raver, Blair y Willoughby, 2013; Blair y Raver, 2012) y de niños con dificultades neuropsicológicas como el Trastorno por Déficit de Atención con/ 
sin Hiperactividad (TDA-H) o psiquiátricas, como los trastornos de la conducta. Por todo lo anterior, aumentan las propuestas de intervención de las FE tanto a nivel clínico como social y educativo. Algunos de los programas son (blog profesor Díaz Atienza):

- Programa cognitivo-conductual de Kendall, Padawer y Zupan (1980).

- Programa en auto instrucciones verbales de Kirby y Grimley (1986).

- Programa de entrenamiento en solución de problemas en grupo de Goldstein y Pollock (1998).

- Programa de entrenamiento en habilidades sociales de Goldstein y Pollack (1988).

- Programa de intervención educativa para aumentar la atención y la flexibilidad de Gargallo (1997).

En resumen, queda patente la importancia del desarrollo de las capacidades de autorregulación para el rendimiento escolar y personal, verdadero reto para los educadores y psicólogos u otros profesionales de la etapa infantil y primaria. Este trabajo propone pues a continuación dos programas de intervención sobre las FE que podrían bien ser aplicados y adaptados al aula por profesionales de la educación o bien empleados por profesionales del ámbito clínico.

\section{OBJETIVO, MÉTODO Y MATERIALES}

El presente trabajo teórico consiste en una revisión bibliográfica -realizada desde una perspectiva integradora de la neuropsicología infantil y la neuroeducación- sobre la estimulación de las FE desde el aula. Pretende proponer una aplicación práctica y participativa de estimulación cognitiva en el aula, en Educación Infantil y Primaria (niños de 10 a 14 años).

La información respecto al tema planteado se ha recopilado mediante la búsqueda y selección de todo tipo de publicaciones (artículos, libros, actas de congresos, blogs) que tratan sobre neuropsicología infantil en el aula en las siguientes bases de datos, entre otras: EBSCO, PSYINFO, PROQUEST y "scholar google", limitando la búsqueda al tema expuesto en este artículo y a artículos lo más recientes posibles. 
El análisis posterior de dichos artículos nos permitió, por una parte, elaborar una síntesis del estado actual de la cuestión para facilitar al lector la comprensión del concepto de Neuroeducación o Neurodidáctica y, por otra, proponer una estrategia y metodología de intervención práctica sobre las FE desde el aula, dando prioridad a dos artículos en particular.

\section{RESULTADOS}

Como bien señala Lussier (2014), en rehabilitación neuropsicológica, la mayoría de los modelos de intervención sobre FE se basan en el concepto de plasticidad cerebral y buscan la restauración o reorganización de las funciones siguiendo generalmente dos tipos de procesos: Ios de abajoarriba (bottom-up strategy) y los de arriba-abajo (top-down strategy) (Robertson y Murre, 1999; Seron, 1984). Por un lado, Ios principios de restauración bottom-up han dado lugar a la aparición de métodos de intervención denominados específicos de dominio, que consisten en realizar un entrenamiento sistemático, intensivo y repetitivo de las FE (Robertson y Murre, 1999). Buen ejemplo de ello serían la rehabilitación cognitiva, las terapias asistidas por ordenador y el neurofeedback. Por otro lado, basándose en que la activación de funciones superiores como las FE tiene una influencia sobre sistemas inferiores que también puede reflejarse en términos de reorganización cortical (Robertson y Murre, 1999), los principios de restauración top-down suelen asociarse a métodos de rehabilitación que se centran en la metacognición y toma de conciencia de los déficits propios para desarrollar y entrenar capacidades y estrategias de autorregulación compensatorias. Los programas de intervención basados en el modelaje, el discurso interno (auto-instrucciones) o las estrategias de resolución de problemas, son un ejemplo de este tipo de método de intervención (Lussier, 2014).

En el ámbito de las estrategias y métodos de intervención top-down, la original propuesta de intervención de Gagné (2004) Ilamada Reflecto, y destacada por Lussier (2014) está pensada para mejorar la capacidad de auto-regulación en niños, consistente en representar y asociar cada una de las funciones cognitivas implicadas en los aprendizajes a graciosos personajes de dibujos animados, que los niños conocen y aprenden a utilizar en diversas situaciones (Gagné y Longprés, 2004).

Inspirándose en el programa de Gagné anterior, Lussier (2014) y su equipo crean el Programa de Intervención sobre las Funciones Atencionales y Meta-cognitivas (PIFAM) inicialmente diseñado para niños con TDA-H. Este programa pretende mejorar el potencial meta-cognitivo de los niños desarrollando y entrenando habilidades de autorregulación comportamental 
y cognitiva (lenguaje interno, control de la impulsividad, resistencia a la distracción, ejecución secuencial, gestión de la información...). Además, el programa también está pensado para promover la adquisición de estrategias de aprendizaje eficaces y su generalización a diferentes contextos: pedagógico, socio-emocional y conductual (Lussier, 2014).

Concretamente, el PIFAM consiste en la realización de talleres grupales semanales a lo largo de un trimestre (12 talleres). Está previsto para niños con edades comprendidas entre 10 y 14 años.

En los talleres se hacen actividades lúdicas donde se solicita a los niños el uso de diversas estrategias de pensamiento reflexivo (toma de conciencia y adopción de estrategias de metacognición y autorregulación) aplicadas a diferentes situaciones de la vida diaria.

El primer taller consiste en una introducción al método y a las reglas y objetivos de los talleres en general: a lo largo de los 12 talleres se harán actividades para que cada niño conozca sus fuerzas y sus debilidades y, cada semana, se propondrá una "misión" y un "desafío" individual para que los niños consoliden y generalicen el trabajo hecho en el taller a su vida diaria, si es posible, con la implicación de sus padres. Por ello, en esta primera sesión los participantes también se comprometen formalmente con el desarrollo del taller, de sus "misiones" y "desafíos" semanales. Tras la introducción, el primer taller tiene como objetivo -siempre mediante actividades lúdicas y participativas- que los niños descubran sus diversas formas de inteligencia y los mecanismos de la cognición. Para ello, se les presenta el funcionamiento del cerebro con material concreto, analogías con personajes (siguiendo el modelo de Gagné que asocia un personaje a una función cognitiva) y experiencias directas (juegos de exploración, por ejemplo). También se les administra un cuestionario que permite identificar fuerzas y debilidades de uno mismo.

En el segundo taller se trabaja una fase importante para el éxito del taller completo: el reconocimiento y la toma de conciencia de las dificultades, así como de sus soluciones, promoviendo la aceptación de los déficits desde un punto de vista constructivo y positivo (los niños notan que tienen el poder de cambiar algunas cosas). La siguiente fase de intervención (talleres 3 y 4) se dedica al entrenamiento de las capacidades de visualización (elaboración de representaciones visuales y creación de imágenes mentales) y verbalización (acceso al léxico, lenguaje descriptivo, desarrollo del lenguaje interno), capacidades extremadamente importantes tanto para la elaboración de representaciones mentales y la capacidad de autorregulación como para el entrenamiento subyacente de las FE. 
Los talleres 5 a 11 se dedican a la comprensión, a la exploración y al entrenamiento de las FE implicadas en los mecanismos de autorregulación del pensamiento, del afecto y del comportamiento. Esto se hace siguiendo la metodología propuesta por Gagné: asociar un personaje animado a cada una de las siguientes funciones cognitivas trabajadas en el siguiente orden: gestión del tiempo y recursos personales (taller 5), atención-concentración (taller 6), estrategias de aprendizaje como clasificación y categorización (taller 7 , flexibilidad cognitiva y creatividad (taller 8), planificación y anticipación (taller 9), tratamiento secuencial o "paso a paso" de la información y realización cuidada y limpia de objetivos (taller 10) y mecanismos de inhibición (taller 11).

El hecho de asociar cada función cognitiva a un personaje cuya profesión forma parte del repertorio léxico de los niños facilita la creación de relaciones conceptuales y, por tanto, la comprensión de lo que son las FE y meta cognitivas en los niños. Así, en el PIFAM se utilizan las siguientes metáforas: el mayordomo para la gestión del tiempo y de los recursos, el detective para la atención, el bibliotecario para la estrategias de aprendizaje, el artista para la flexibilidad y creatividad, el arquitecto para la planificación y anticipación, el carpintero para el tratamiento secuencial de la información y realización de objetivos, y el controlador aéreo para la inhibición. La presentación de los temas y actividades de cada taller se completa con ejercicios variados que fomentan la adquisición de estrategias de gestión mental.

Además, para permitir la generalización de lo aprendido en el taller a las actividades de la vida cotidiana, cada semana la profesional que dirige el taller resalta la relación entre la habilidad cognitiva trabajada en esa sesión y su carácter o implicación pedagógica, conductual o socio-emocional. En este sentido, el taller también incluye un modulo de exploración de aptitudes sociales, que se realizará de manera transversal, como el resto del taller, ya que se basa en la participación activa de los niños en las actividades, el trabajo en equipo (incluyendo, a veces, nociones de competitividad), la propuesta de discusiones y reflexiones grupales, el método lúdico (aprendizaje mediante el juego), la motivación y la transferencia de conocimientos y competencias al plano personal, socio-emocional y pedagógico.

La última sesión de intervención (taller 12) tiene como objetivo la generalización de las habilidades adquiridas en los talleres anteriores a contextos más amplios, variados y menos estructurados: los participantes deben realizar un proyecto colectivo (maqueta, poster, juego...) que represente su experiencia con los talleres. Además, al final del taller, los profesionales encargados del mismo mantienen una breve reunión (20 minutos) con los padres -que ya conocen de las reuniones de feedback semanal al final de cada sesión- para informarles de la evolución general del niño a lo largo de los 3 
meses de intervención. Este seguimiento individualizado y la transmisión de dicha información (implicación, fuerzas y debilidades del niño) a los padres se hacen usando la hoja de seguimiento semanal que cada terapeuta rellena sobre cada niño en cada sesión.

El bajo ratio paciente/terapeutas (2:1) -propuesto por Lussier en el PIFAM y susceptible de ser modificado en ámbito educativo- permite un estrecho seguimiento de la evolución y acompañamiento de cada participante en sus aprendizajes. Así, para asegurar el poder explicativo de las metáforas entre funciones cognitivas y personajes y la consecuente creación de esquemas cognitivos al respecto, las profesionales utilizarán de manera sistemática y repetida la referencia a los personajes y sus funciones en diferentes contextos (pedagógico, social, emocional y conductual). Para promover el uso más sistemático posible de la reflexión interior, antes de comenzar una actividad o situación, las profesionales a cargo del taller también emplearán métodos de aprendizaje por modelado y, sobre todo, por auto-instrucción, así como el refuerzo positivo y técnicas de modificación de conducta (registros o autoregistros, contratos de conducta, premios...) usados como instrumentos de medida externos o internos y técnicas de refuerzo y recompensa.

Siguiendo a Lussier (2014), se podría entonces afirmar que intervenir en las funciones meta-cognitivas superiores (estrategia top-down) y de manera lúdica y ecológica, tal como lo hace el PIFAM, mejora sensiblemente las funciones cognitivas ejecutivas y atencionales. Sin embargo, estas conclusiones -hechas a partir de la observación y la recogida de comentarios de padres a lo largo 10 años de experiencia clínica con el PIFAM-, siguen necesitando aún mayor evidencia científica.

En efecto, si bien la evidencia clínica de la eficacia de las estrategias de intervención -según el modelo top-down les parece clara a numerosos autores (Lussier, 2004, Diamond, 2007), carece todavía de la suficiente evidencia científica para otros. En este sentido, el trabajo de Baron (2017) revisa y sintetiza de forma exhaustiva y sistemática la evidencia científica del programa de intervención Tools of the Mind para la estimulación de las habilidades de autorregulación y académicas: incluye tamaños de efectos cuantitativos y emplea mecanismos estadísticos para controlar posibles confusiones. Siempre dentro del marco teórico anterior -centrado en mejorar las capacidades de autorregulación, las habilidades pedagógicas y socio-emocionales desde contextos educativos formales-, este modelo de intervención complementa el anterior de Lussier (PIFAM) por poder aplicarse en nivel preescolar. El programa de intervención Tools of the Mind para el aula, inicialmente propuesto por la psicología rusa, retomado y adaptado después por Diamond (2007) y recientemente validado por Baron (2017) tiene como objetivo potenciar las capacidades de autorregulación y otras habilidades socio-emocionales 
y académicas, por lo que se ha implementado notablemente su uso en las clases de los EEUU, Canadá y Chile. La revisión de Baron (2017) evidencia beneficios estadísticamente significativos del uso del programa en matemáticas y los resultados para autorregulación y alfabetización son favorables al programa, aunque no alcanzan niveles de significación estadística.

Como señala Baron (2017), la teoría del desarrollo de Vygotsky es central en el enfoque del programa Tools of the Mind y dicho programa está arraigado en la investigación neuropsicológica sobre el desarrollo de autorregulación y FE en niños (Bodrova y Leong, 2015; Diamond, 2007). A diferencia de otras propuestas de intervención en autorregulación, que a menudo implican planes individualizados para poblaciones de niños específicos (Gulchak, 2008; Soares y cols., 2009) o series de ejercicios para complementar un plan de estudios existente (Bierman y cols., 2007), Tools of the Mind pretende ser un plan de estudios integral diseñado para todos los niños y las partes del currículo.

Como el taller anterior, utiliza el juego imaginario como forma de comprensión y trabajo sobre los mecanismos de autorregulación: los escenarios de juego requieren de los niños que recuerden su papel de fantasía y lo actúen (memoria de trabajo), inhiban el impulso al cambiar roles arbitrariamente (control inhibitorio) y intercambien de forma flexible sus personalidades y las del rol que han asumido (flexibilidad cognitiva). Para transformar a los niños en "maestros de sus propias conductas" -como decía Vygotsky- el programa se basa en tres elementos: primero el docente regula a los estudiantes, segundo los estudiantes se regulan entre ellos y por último, los estudiantes se auto-regulan. Es decir que el aprendizaje consciente de la capacidad para regular pensamientos, emociones y acciones internas comienza mediado externamente (por un adulto o un compañero más competente). (Bodrova y Leong, 2007). Además, el programa integra las actividades orientadas a la autorregulación dentro de la instrucción académica (Bodrova y Leong, 2011): cada actividad contiene tanto una habilidad académica meta (por ejemplo, leer un libro con un compañero de clase) como una capacidad de autorregulación meta (esperar el turno de leer el libro).

En general, el programa incluye más de 60 actividades de alfabetización, matemáticas o situaciones de juego y está diseñado para ser implementado por los maestros durante un año académico completo (Bodrova y Leong, 2011). 


\section{DISCUSIÓN-CONCLUSIÓN}

Además de responder a la actual demanda sobre cómo el conocimiento del funcionamiento cerebral puede mejorar los aprendizajes, la aportación particular de este trabajo es su naturaleza integral, es decir que su contenido puede aplicarse a todos los niños del ámbito educativo de Educación Infantil y Primaria, así como a la intervención específica psicopedagógica en el colegio o neuropsicológica extraescolar, en niños con dificultades de autorregulación.

Sin embargo, somos conscientes que el reducido número de estudios elegidos para realizar este trabajo constituye una limitación importante. No obstante, los resultados y conclusiones son consistentes con la escasa literatura actual sobre otros programas de autorregulación en niños. En este sentido, la revisión sistemática reciente de las intervenciones en FE en la primera infancia de Jacob y Parkinson (2015) también informó de dificultades para identificar gran cantidad de estudios. Por lo tanto, el pequeño número de estudios incluidos en esta revisión refleja principalmente la escueta base de evidencia observada en este ámbito de intervención.

En conclusión, los dos programas de intervención en FE presentados en el presente trabajo se alinean con las teorías del desarrollo infantil más 40 actuales en neuropsicología infantil. Si bien la implementación en contexto clínico y educativo de la Neuroeducación y estimulación de las FE desde el aula todavía está por desarrollarse, la información aportada por este trabajo permite tanto a los educadores como a los clínicos, disponer de información y estrategias eficaces para poder plantear objetivos y actividades educativas que mejoren las FE y el rendimiento académico.

\section{BIBLIOGRAFÍA}

Abad, S., Brusasca, M. C. y Labiano, L. M. (2009). Neuropsicología infantil y educación especial. Revista Intercontinental de Psicología y Educación, 11(1) 199-216.

Alexander, K. L., Entwistle, D. R., Horsey, C. S., Kabbani, N. S., Atkinson, M., Halsey, K. y Kinder, K. (2001). Schulabsentismus und Dropout. Teachers College Record, 70, 87-107.

Anderson, P. (2002). Assessment and Development of Executive Function (EF) During Childhood. Child Neuropsychology, 8, 71-82.

Anderson, V., Northam, E. y Wrennall, J. (2017). Developmental neuropsychology: A clinical approach. Routledge.

Ardila, A., Rosselli, M. y Villaseñor, E. M. (2005). Neuropsicología de los trastornos del aprendizaje. UNAM. 
Ávila Matamoros, A. M. y Cuervo Martínez, L. Á. (2010). Adaptación del cuestionario de madurez neuropsicológica infantil Cumanin de Portellano.

Barkley, R. A. (2001). The executive functions and self-regulation: An evolutionary neuropsychological perspective. Neuropsychology review, 11(1), 1-29.

Barnett, M. L. y King, A. A. (2008). Good fences make good neighbors: A longitudinal analysis of an industry self-regulatory institution. Academy of Management Journal, 51(6), 1150-1170.

Baron, A., Evangelou, M., Malmberg, L. E., Melendez-Torres, G. J., Baron, A. y Gardens, N. (2017). The Tools of the Mind curriculum for improving self-regulation in early childhood: a systematic review. Campbell Systematic Reviews.

Bastién, G. M., Mora, C. y Sánchez, D. (2013). Obstáculos en la resolución de problemas en alumnos de bajo rendimiento.

Bell, V. (2013). Enciclopedia sobre el Desarrollo en la primera infancia. GRIP-Université de Montréal.

Bierman, K. L., Domitrovich, C. E., Nix, R. L., Gest, S. D., Welsh, J. A., Greenberg, M. T. y Gill, S. (2008). Promoting academic and social emotional school readiness: The Head Start REDI program. Child development, 79(6), 1802-1817.

Blair, C. y Raver, C. C. (2012). Child development in the context of adversity: experiential canalization of brain and behavior. American Psychologist, 67(4), 309.

Blair, C. y Ursache, A. (2011). A bidirectional model of executive functions and selfregulation. Handbook of self-regulation: Research, theory, and applications, 2, 300-320.

Blair, C., Zelazo, P. D. y Greenberg, M. T. (2005). Measurement of Executive Function in Early Childhood: A Special Issue of Developmental Neuropsychology. Psychology Press.

Bodrova, E. y Leong, D. (2007). Tools of the mind: The Vygotskian approach to early childhood education.

Bodrova, E., Leong, D. J., \& Akhutina, T. V. (2011). When everything new is wellforgotten old: Vygotsky/Luria insights in the development of executive functions. New Directions for Child and Adolescent Development, (133), 11-28.

Campos, A. (2010). Neuroeducación: uniendo las neurociencias y la educación en la búsqueda del desarrollo humano. La Educación. Revista Digital, 143, 1-14.

Diamond, A. (2014). Understanding executive functions: What helps or hinders them and how executive functions and language development mutually support one another. Perspectives on Language and Literacy, 4O(2), 7.

Diamond, A., Barnett, W. S., Thomas, J., \& Munro, S. (2007). Preschool program improves cognitive control. Science (New York, NY), 318(5855), 1387.

Gagné, P.P. y Longpré, L.P. (2004). Apprendre...avec Réflecto. Chenelière McGraw-Hill.

García, D. y Muñoz, P. (2000). Funciones ejecutivas y rendimiento escolar en educación primaria. Un estudio exploratorio. Revista complutense de educación, 11(1), 39-56.

Goswami, U. (2006). Neuroscience and education: from research to practice?. Nature reviews neuroscience, 7(5), 406. 
Goswami, U. (2009). Mind, brain, and literacy: Biomarkers as usable knowledge for education. Mind, Brain, and Education, 3(3), 176-184.

Gulchak, D. J. (2008). Using a mobile handheld computer to teach a student with an emotional and behavioral disorder to self-monitor attention. Education and Treatment of Children, 31(4), 567-581.

Hongwanishkul, D., Happaney, K. R., Lee, W. S., \& Zelazo, P. D. (2005). Assessment of hot and cool executive function in young children: Age-related changes and individual differences. Developmental neuropsychology, 28(2), 617-644.

Jacob, R. y Parkinson, J. (2015). The potential for school-based interventions that target executive function to improve academic achievement: A review. Review of Educational Research, 85(4), 512-552.

Kennard, M. A. (1942). Cortical reorganization of motor function. Archives of Neurology and Psychiatry, 48, 227-240.

Lanoë, C., Rossi, S., Froment, L. y Lubin, A. (2015). Le programme pédagogique neuroéducatif "À la découverte de mon cerveau»: quels bénéfices pour les élèves d'école élémentaire?. Approche neuropsychologique des apprentissages chez l'enfant, 134, $55-45$

Lussier, F (2014). Programme d'intervention auprès des enfants qui souffrent de TDA/H. Psychologie Québec, 31(03).

Manga, D. y Ramos, F. (1999). Evaluación neuropsicológica. Clínica y Salud, 3, 331-376.

Manga, D. y Ramos, F. (2001). Evaluación de los síndromes neuropsicológicos infantiles. Revista de Neurología, 32(7), 664-675.

Manga, D. y Ramos, F. (2017). El legado de Luria y la neuropsicología escolar. Psychology, Society, \& Education, 3(1), 1-13.

Meltzer, L. (Ed.). (2018). Executive function in education: From theory to practice. Guilford Publications.

O'Shaughnessy, T. E., Lane, K. L., Gresham, F. M. y Beebe-Frankenberger, M. E. (2003). Children placed at risk for learning and behavioral difficulties: Implementing a school-wide system of early identification and intervention. Remedial and Special Education, 24(1), 27-35.

Raver, C. C., Blair, C. y Willoughby, M. (2013). Poverty as a predictor of 4-year-olds' executive function: New perspectives on models of differential susceptibility. Developmental psychology, 49(2), 292.

Robertson, I. H. y Murre, J. M. (1999). Rehabilitation of brain damage: Brain plasticity and principles of guided recovery. Psychological bulletin, 125(5), 544.

Seron, X. (1984). Reeducation strategies in neuropsychology: cognitive and pragmatic approaches. Advances in Neurology, 42, 317-325.

Tapia, A., Anchatuña, A., Cueva, M., Poma, R., Jiménez, S. y Corrales, E. (2017). Las neurociencias. Una visión de su aplicación en la educación. Open Journal Systems en Revista: Revista de entrenamiento, 4(1), 61-74.

Zelazo, P. D. y Müller, U. (2002). Executive function in typical and atypical development. Blackwell handbook of childhood cognitive development, 445-469. 